\title{
A comparison of monodomain and bidomain reaction-diffusion models for action potential propagation in the human heart
}

\author{
Mark Potse, Bruno Dubé, Jacques Richer, Alain Vinet, Member, IEEE \\ and Ramesh M. Gulrajani Fellow, IEEE
}

\begin{abstract}
A bidomain reaction-diffusion model of the human heart was developed, and potentials resulting from normal depolarization and repolarization were compared with results from a compatible monodomain model. Comparisons were made for an empty isolated heart and for a heart with fluid-filled ventricles. Both sinus rhythm and ectopic activation were simulated. The bidomain model took 2 days on 32 processors to simulate a complete cardiac cycle. Differences between monodomain and bidomain results were extremely small, even for the extracellular potentials, which in case of the monodomain model were computed with a high-resolution forward model. Propagation of activation was $2 \%$ faster in the bidomain model than in the monodomain model. Electrograms computed with monodomain and bidomain models were visually indistinguishable. We conclude that, in the absence of applied currents, propagating action potentials on the scale of a human heart can be studied with a monodomain model.
\end{abstract}

Index Terms-Cardiac membrane model, bidomain model, monodomain model, computer heart model

This is a preprint prepared by the authors. It was published as Potse M, Dubé B, Richer J, Vinet A, and Gulrajani RM. A comparison of monodomain and bidomain reaction-diffusion models for action potential propagation in the human heart. IEEE Trans. Biomed. Eng., 53:2425-2435, 2006.

doi:10.1109/TBME.2006.880875

Manuscript submitted November 3, 2005; accepted May 21, 2006. This work was supported by the Natural Sciences and Engineering Research Council of Canada. M. Potse was supported by FRSQ, Québec, and The Netherlands Organization for Scientific Research (NWO).

M. Potse is with the Department of Physiology, Institute of Biomedical Engineering, Université de Montréal, PO Box 6128, Station Centre-ville, Montréal, QC H3C 3J7 Canada, and Sacré-Coeur hospital, Montréal, QC H4J 1C5 Canada (phone: +1-514-338 2222 \#2519, fax: +1-514-338 2694, e-mail: mark@potse.nl).

B. Dubé is with the Department of Physiology, Institute of Biomedical Engineering, Université de Montréal, Montréal, QC H3C 3J7 Canada, and Sacré-Coeur hospital, Montréal, QC H4J 1C5 Canada.

J. Richer is with the Réseau québécois de calcul de haute performance, Montréal, QC H3C 3J7 Canada.

A. Vinet is with the Department of Physiology, Institute of Biomedical Engineering, Université de Montréal, Montréal, QC H3C 3J7 Canada, and Sacré-Coeur hospital, Montréal, QC H4J 1C5 Canada.

R. M. Gulrajani, deceased, was with the Department of Physiology, Institute of Biomedical Engineering, Université de Montréal, Montréal, QC H3C 3J7 Canada, and Sacré-Coeur hospital, Montréal, QC H4J 1C5 Canada.

Copyright (c) 2006 IEEE. Personal use of this material is permitted. However, permission to use this material for any other purposes must be obtained from the IEEE by sending an email to pubs-permissions@ieee.org.

\section{INTRODUCTION}

$\mathbf{T}$ HE bidomain model represents active myocardium on a macroscopic scale by relating membrane ionic current, membrane potential $\left(V_{\mathrm{m}}\right)$, and extracellular potential $\left(\phi_{\mathrm{e}}\right)[1]$. Conceived in 1969 [2] and first expressed formally in 1978 [3], [4], the bidomain model was initially used to derive forward models, which compute extracellular and body-surface potentials from given membrane potentials [4]-[7]. Later the bidomain model was used to link multiple membrane models together to form a bidomain reaction-diffusion ( $\mathrm{R}-\mathrm{D}$ ) model [8], [9], which simulates propagating activation based on no other premises than those of the membrane model, those of the bidomain model, and Maxwell's equations.

Bidomain R-D models have to solve an implicit equation to obtain $\phi_{\mathrm{e}}$ at every simulated time instant. Therefore their use has long been limited to relatively small preparations. For human-heart simulations, either fixed velocities and waveforms were used or the monodomain approximation was made to keep the problem tractable [10]-[16]. The monodomain approximation has allowed the development of R-D-type models for the human heart as early as in 1991 [10], and produces realistic activation patterns and $V_{\mathrm{m}}$. However, it cannot be applied in all situations because it does not permit currents in the extracellular domain to influence $V_{\mathrm{m}}$ and ionic currents. This influence obviously has to be taken into account when there are applied currents due to pacing or defibrillation [17]-[20]. But even when there are no impressed currents, the current flow through the extracellular and extracardiac domains may influence cardiac sources in a way that can only be represented by a bidomain model [9], [21]-[23].

The accuracy of a monodomain model can be tested by comparing results with a bidomain model. Whole-heart bidomain R-D models for small species such as the mouse and the rabbit have recently been reported [24], [25]. Several groups have also described methods designed for whole-heart studies and tested them on a smaller scale [22], [23], [26]. We developed such a model for the human heart. Our model differs from previous animal heart models with respect to its scale and its specifically human parameters, and from previous human heart models because of its ability to simulate a complete cardiac cycle in a reasonable period of time.

The purpose of our study was 1) to develop a bidomain human heart model and 2) to test the appropriateness of the monodomain approximation to simulate propagation in the 
TABLE I

MEDIA, MATERIALS, AND CONDUCTIVITY VALUES $\left(\mathrm{SM}^{-1}\right)$

\begin{tabular}{llcccc}
\hline medium & material & $\sigma_{\mathrm{eT}}$ & $\sigma_{\mathrm{eL}}$ & $\sigma_{\mathrm{iT}}$ & $\sigma_{\mathrm{iL}}$ \\
\hline active & ventricular muscle & 0.12 & 0.30 & 0.03 & 0.30 \\
conductive & connective tissue & 0.20 & 0.20 & 0 & 0 \\
& fluid & 0.60 & 0.60 & 0 & 0 \\
insulating & air & 0 & 0 & 0 & 0 \\
\hline
\end{tabular}

human heart. To test the monodomain model, we compared conduction velocities and $\phi_{\mathrm{e}}$ between monodomain and bidomain models. To obtain $\phi_{\mathrm{e}}$ for the monodomain model we used a forward model that computed $\phi_{\mathrm{e}}$ in exactly the same way as the bidomain model. The comparisons were done for an isolated-heart model, and for one in which the ventricular cavities were filled with fluid.

\section{Methods}

We consider two domains: intracellular and extracellular. The intracellular domain exists only for active tissue. The extracellular domain exists in active tissue as well as in other tissue and in surrounding fluid. Three media are distinguished based on the conductivity in the two domains: 1) "Active tissue" is conductive in both domains; $V_{\mathrm{m}}$ and $\phi_{\mathrm{e}}$ are defined in this medium. 2) "Conductive medium" conducts only in the extracellular domain; consequently only $\phi_{\mathrm{e}}$ is defined. 3) "Insulating medium" is nonconductive in both domains; neither potential is defined for it. There are several materials, each belonging to one medium, and each having different conductivity. Boundary conditions must be considered between media but not between materials, as discussed below. An overview of media and materials, together with their conductivities, is given in Tab. I.

\section{A. Anatomic Model}

The model anatomy was based on CT data of a human heart obtained at autopsy [27]. Muscle and connective tissue were previously identified in the CT slices at 1-mm resolution. From these data we made a surface description, which was subsequently used to determine the node types in a threedimensional (3-D) finite-difference (FD) grid with 0.2-mm resolution $(567 \times 501 \times 711$ nodes $)$. Nodes could represent ventricular muscle $(13 \%)$, atrial muscle or connective tissue (5\%); fluid (4-10\%); or air (73-78\%). We will use the term "node" for those grid points that represent tissue or fluid. The atria were not activated in this study, but were retained in order to allow the simulation of a grounding electrode on the right atrium, similar to that used in isolated Langendorffperfused hearts. Therefore we replaced the atrial muscle by connective tissue (Tab. I). Ventricular muscle was represented by an inhomogeneously anisotropic bidomain.

For each ventricular node, we determined a minimal distance to endocardium and epicardium $\left(d_{\text {endo }}, d_{\text {epi }}\right)$ using a 3 -D distance transform algorithm with $(1, \sqrt{2}, \sqrt{3})$ chamfer distances [28]. A thickness parameter $e$ was defined as

$$
e=d_{\text {endo }} /\left(d_{\text {endo }}+d_{\text {epi }}\right) \text {. }
$$

The local fiber orientations were made orthogonal to the vector $\nabla \bar{e}$, where each element of $\bar{e}$ is the average of the corresponding element of $e$ and its 26 neighbors. In practice, $\nabla \bar{e}$ did not suffer from singularities as $\nabla e$ would. This method ensured alignment of the fibers with the cardiac surfaces together with smoothly varying angles (Fig. 1). The fiber orientation was further determined by a helix angle

$$
\alpha=R(1-2 e)^{3}
$$

to account for transmurally rotating fiber orientation [29], [30]. We set $R$ equal to $\pi / 3$ for the left ventricle (LV) and $\pi / 4$ for the right ventricle (RV). The expression for $\alpha$ was adapted from one by Beyar and Sideman [31], which was based on measurements by Streeter et al. [29]. The power 3 served to match better the nonlinear curve that Streeter et al. found for individual cases. The parameter $e$ was also used to position three layers of different ventricular cell types: epicardial cells in the outer $20 \%$ of the wall, M-cells in the next $30 \%$, and endocardial cells in the inner $50 \%$ [32], [33]. Ionic currents $I_{\text {ion }}$ were computed using the model of the human ventricular cell membrane formulated by Bernus et al. [34], which accounts for these three cell types. We assumed that the interventricular septum is layered like the LV wall, except for the presence of endocardial cells on the RV side. We ignored differences between LV and RV cells [35], [36]. The different cell layers are illustrated in Fig. 1. The model has no Purkinje network; for sinus rhythm simulation we stimulated the ventricles at the early activation sites and corresponding times published by Durrer et al. [27], [37]. Stimulation was performed by transmembrane current injection $\left(200 \mu \mathrm{A} / \mathrm{cm}^{2}\right.$ during $2 \mathrm{~ms}$, interrupted when $V_{\mathrm{m}}$ exceeded $30 \mathrm{mV}$ ) to mimic a physiological stimulus. The same stimulation technique was used to initiate ectopic activation.

\section{B. Bidomain Model}

The bidomain model [2]-[4] describes the cardiac tissue as consisting of two co-located syncytia termed the intracellular and extracellular domain, which are characterized by conductivity tensors $G_{\mathrm{i}}$ and $G_{\mathrm{e}}$, respectively. A current with surface density $I_{\mathrm{m}}$ flows between the two domains. The intracellular and extracellular potentials $\phi_{\mathrm{i}}$ and $\phi_{\mathrm{e}}$ are subject to the bidomain equations:

$$
\begin{aligned}
\nabla \cdot\left(G_{\mathrm{i}} \nabla \phi_{\mathrm{i}}\right) & =\beta I_{\mathrm{m}} \\
\nabla \cdot\left(G_{\mathrm{e}} \nabla \phi_{\mathrm{e}}\right) & =-\beta I_{\mathrm{m}}
\end{aligned}
$$

where $\beta$ is the membrane surface-to-volume ratio, i.e. the amount of membrane found in a given volume of tissue. The transmembrane current density $I_{\mathrm{m}}$ consists of a capacitive part, an ionic part $I_{\text {ion }}\left(V_{\mathrm{m}}, t\right)$ generated by the cell membrane, and an imposed stimulation current density $I_{\mathrm{S}}$ :

$$
I_{\mathrm{m}}=C_{\mathrm{m}} \frac{\partial V_{\mathrm{m}}}{\partial t}+I_{\text {ion }}+I_{\mathrm{s}}
$$

where $C_{\mathrm{m}}$ is the membrane capacitance per unit area. We used $\beta=1000 \mathrm{~cm}^{-1}$ and $C_{\mathrm{m}}=1 \mu \mathrm{F} / \mathrm{cm}^{2}$ (see Discussion). Using 


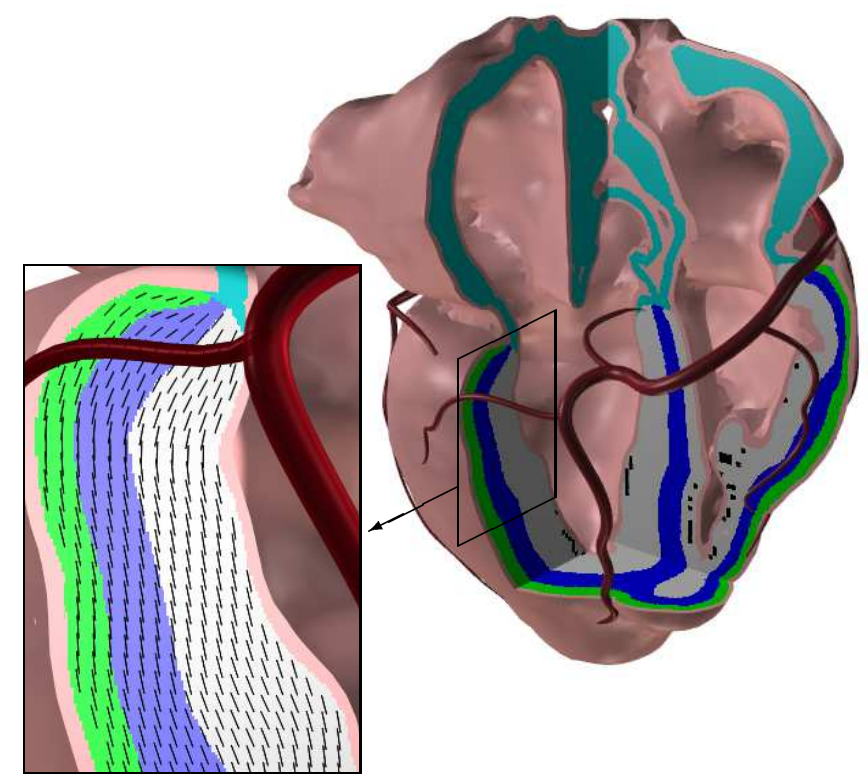

Fig. 1. Top right: heart model showing the tissue embedded in a thin layer of fluid (pink). Tissue types are identified with colors: gray for endocardial, blue for M-cells, green for epicardial, and cyan for connective tissue. Black spots indicate early activation sites, which play the role of Purkinje-muscle junctions in our model. The heart is shown approximately in a posterior view with the left ventricular cavity on the left. Between the atria, the aortic annulus is visible. Coronary arteries are shown for orientation (veins were omitted); most prominent is the right circumflex artery which bends down in the crux cordis. Bottom left: detail of the left ventricular wall showing, at 1-mm resolution, the planes in which the fibers lie; the helix angle $\alpha$ is not shown.

an operator splitting approach [38] we write the equations in terms of $\phi_{\mathrm{e}}$ and $V_{\mathrm{m}}$ as an R-D equation

$$
\frac{\partial V_{\mathrm{m}}}{\partial t}=\frac{1}{\beta C_{\mathrm{m}}}\left\{\nabla \cdot\left(G_{\mathrm{i}} \nabla\left(V_{\mathrm{m}}+\phi_{\mathrm{e}}\right)\right)-\beta\left(I_{\mathrm{ion}}+I_{\mathrm{s}}\right)\right\},
$$

which we used to integrate $V_{\mathrm{m}}$ over time, and an implicit equation

$$
\nabla \cdot\left(\left(G_{\mathrm{i}}+G_{\mathrm{e}}\right) \nabla \phi_{\mathrm{e}}\right)=-\nabla \cdot\left(G_{\mathrm{i}} \nabla V_{\mathrm{m}}\right)
$$

from which we solved $\phi_{\mathrm{e}}$.

\section{Implementation}

Computations were performed on 32 processors of an SGI Altix 3700 computer. This system has 64 nodes, each containing 2 processors and $8 \mathrm{~GB}$ of memory. The nodes intercommunicate over $3.2 \mathrm{~GB} / \mathrm{s}$ (bidirectional) links. Although its memory is decentralized, the system employs a single memory image. The program was written in $\mathrm{C}$ and parallelized with OpenMP. Care was taken to place each data element on the node where it would be used most often, and to minimize sharing of data between nodes, since this minimizes memory access times. We also maximized cache line re-use by storing large arrays exactly in the order in which they were used by critical sections of the program.

Equations (6) and (7) were discretized using a forward Euler approach to integrate over time, resulting in

$$
V_{\mathrm{m}}^{t+\delta t}=V_{\mathrm{m}}^{t}+\frac{\delta t}{\beta C_{\mathrm{m}}}\left\{A \cdot\left(V_{\mathrm{m}}^{t}+\phi_{\mathrm{e}}^{t}\right)-\beta\left(I_{\text {ion }}^{t}+I_{\mathrm{s}}^{t}\right)\right\}
$$

and

$$
B \cdot \phi_{\mathrm{e}}^{t+\delta t}=A \cdot V_{\mathrm{m}}^{t+\delta t}
$$

where $A$ and $B$ are $N \times N$ matrices whose coefficients are computed from $G_{\mathrm{e}}+G_{\mathrm{i}}$ and $G_{\mathrm{i}}$, respectively; $N$ is the number of nodes. Since the fiber direction was a function of position, $G_{\mathrm{e}}$ and $G_{\mathrm{i}}$ were inhomogeneous tensor fields, which were determined and stored for each FD node by transforming a diagonal conductivity tensor $\operatorname{diag}\left(\sigma_{\mathrm{T}}, \sigma_{\mathrm{T}}, \sigma_{\mathrm{L}}\right)$ from local coordinates that are aligned with the local fiber direction into global coordinates. The $\sigma_{\mathrm{T}}$ and $\sigma_{\mathrm{L}}$ stand for the transverse and longitudinal conductivity, respectively. We used the nominal values determined in a review of experimental studies by Roth [39]. An overview of all conductivity values in myocardium, fluid, and connective tissue is given in Tab. I. We indicate the conductivities by $\sigma_{\mathrm{eT}}, \sigma_{\mathrm{eL}}, \sigma_{\mathrm{iT}}$ and $\sigma_{\mathrm{iL}}$ with subscript 'e' for extracellular, ' $\mathrm{i}$ ' for intracellular, ' $\mathrm{T}$ ' for transverse, and ' $\mathrm{L}$ ' for longitudinal.

The $A$ and $B$ matrices have 19 nonzero elements per row. Since the nonzero positions could be computed efficiently, we stored only the nonzero values, as single-precision floating point numbers. The diagonal elements, whose values must accurately equal the negative sum of the other elements on a row, were computed on the fly and in double precision when a matrix multiplication was performed. This is more efficient than obtaining them from memory. We computed $A$ and $B$ using an expression proposed by Saleheen and $\mathrm{Ng}$, which accounts for the inhomogeneously anisotropic conductivity and allows discontinuities in the conductivity tensor fields [40]. In their approach, the boundary of a medium is just another discontinuity, where $G=\underline{0}$ outside the medium. Since the conductivity outside the medium is zero, there is no current flow across the boundary, so the conditions

$$
\begin{array}{rll}
\hat{n} \cdot G_{\mathrm{i}} \nabla \phi_{\mathrm{i}} & =0 & \text { on } \partial_{\mathrm{tissue}} \\
\hat{n} \cdot G_{\mathrm{e}} \nabla \phi_{\mathrm{e}} & =0 & \text { on } \partial_{\text {air }}
\end{array}
$$

were satisfied without being explicitly enforced. Neighbors outside the simulated volume were treated as air, so that there was also no current flow through this boundary.

In Saleheen and Ng's method [40], the conductivity is constant on "elements" and changes only in a thin transition layer between the elements. An element is a rectangular volume cornered by 8 nodes. The custom-made software that produced the grid ensured that the node types were consistent with the elements (Fig. 2). Because the conductivity is defined on elements rather than nodes, the boundary is infinitesimally close to the nodes (due to the transition layer the boundary does not run through the nodes [40]). Nodes on the tissue boundary therefore represented only a part of the tissue volume represented by interior nodes (Fig. 2). This was accounted for by assigning them a proportional fraction of the standard value for $\beta$, as illustrated in Fig. 2.

At each time step, $I_{\text {ion }}$ was computed from $V_{\mathrm{m}}$ at the previous time step using the membrane model [34]. A "hybrid" method was used to integrate the state variables [41], [42]. The new $V_{\mathrm{m}}$ was then computed by evaluation of (8), and used to compute $\phi_{\mathrm{e}}$ using (9). 


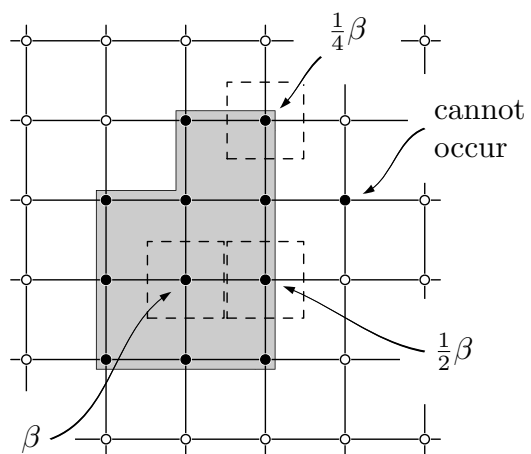

Fig. 2. Finite-difference grid illustrated in 2-D. The gray area represents one of the 3 media in one of the 2 domains, e.g. active tissue in the intracellular domain. Filled circles represent grid nodes that are internal to the medium, open circles nodes that are external. Grid squares correspond to "elements" in Saleheen and Ng's method; only elements that are completely inside the medium have nonzero conductivity. Dashed squares indicate the areas represented by three example nodes: one inside the medium, which has the standard $\beta$ value; one on a boundary, with $\beta / 2$; and one on a corner, with $\beta / 4$. Internal nodes cannot occur as pseudopods, without a neighboring internal element.

Equation (9) is a system of $N$ linear equations. Such systems can be solved with standard software libraries if $N$ is sufficiently small. In our case, where $N \sim 10^{7}$, custom routines proved to be necessary. Classical elimination techniques and matrix decompositions cannot be used because they have memory usage and operations count up to order $N^{2}$. Only iterative methods can solve systems of this size [38]. After initial experiments with the well-known conjugate gradient and biconjugate gradient algorithms [43] and the biconjugate gradient squared variant [44], we finally implemented a more sophisticated biconjugate gradient stabilized (BICGSTAB) solver [45], [46]. This algorithm proved to be the most efficient in terms of CPU time. Application of more recent variants such as QMRCGSTAB [47] gave no further improvement.

The solver routine uses an initial guess for the solution. It is not surprising that $\phi_{\mathrm{e}}^{t}$ is a good initial guess for $\phi_{\mathrm{e}}^{t+\delta t}$, capable of reducing the number of iterations by two orders of magnitude compared to zero initialization. A further reduction was obtained by using

$$
\psi_{1}^{t+\delta t}=\phi_{\mathrm{e}}^{t}+\left(V_{\mathrm{m}}^{t+\delta t}-V_{\mathrm{m}}^{t}\right) k
$$

as a predictor for $\phi_{\mathrm{e}}^{t+\delta t}$, where $k$ is a constant. If the monodomain approximation $G_{\mathrm{i}}=\xi G_{\mathrm{e}}$ [14] for a scalar $\xi$ applies, then $\phi_{\mathrm{e}} \equiv-\xi /(1+\xi) V_{\mathrm{m}}+\phi_{0}$, where $\phi_{0}$ is a constant offset potential, so setting $k=-\xi /(1+\xi)$ would result in perfect prediction. In the general bidomain case, where there is no simple relation between $\phi_{\mathrm{e}}$ and $V_{\mathrm{m}}$, we found that a good prediction can still be obtained with $k=\sigma_{\mathrm{iT}} /\left(\sigma_{\mathrm{iT}}+\sigma_{\mathrm{eT}}\right)$. An alternative predictor is

$$
\psi_{2}^{t+\delta t}=2 \phi_{\mathrm{e}}^{t}-\phi_{\mathrm{e}}^{t-\delta t}
$$

which is obtained by hypothesizing that $\partial^{2} \phi_{\mathrm{e}} / \partial t^{2} \approx 0$ [48]. The reduction of the iteration count obtained with $\psi_{1}$ and $\psi_{2}$ depends on the anisotropy, the complexity of the activation front, and the interval of $\phi_{\mathrm{e}}$ computation. As discussed below, we computed $\phi_{\mathrm{e}}$ at intervals of $50 \mu \mathrm{s}$. For such large steps $\psi_{1}$ performed better, while $\psi_{2}$ was generally better at intervals below $10 \mu \mathrm{s}$.

Iteration of the solver for (9) was terminated when the norm of the residual became smaller than either 1) $10^{-4}$ times the norm of the right-hand side $A \cdot V_{\mathrm{m}}^{t}$, or 2) a fixed threshold of $0.1\left[(\delta x / 0.2 \mathrm{~mm})^{3} N\right]^{1 / 2} \mu \mathrm{A} / \mathrm{cm}^{2}$, where $N$ is the number of nodes and $\delta x$ the spatial step $(\delta x=0.2 \mathrm{~mm}$ for the complete model and $\delta x=0.1 \mathrm{~mm}$ for a small tissue block as discussed below). For comparison, the peak membrane current density associated with a depolarization wavefront is in the order of $10^{5} \mu \mathrm{A} / \mathrm{cm}^{2}$. The fixed-threshold criterion prevents unnecessary accuracy in the repolarization phase. As discussed below, both thresholds were taken 10 times smaller for output of signals, once per millisecond of simulated time.

We used an incomplete-LU (ILU) preconditioner [44], [46], which was parallelized using domain decomposition [46]. Its performance was compared to a diagonal or "Jacobi" preconditioner [43].

The ILU factorization and the $A$ and $B$ matrices together used about half the total memory of the program. The remaining memory was used primarily by the membrane status variables, $V_{\mathrm{m}}, \phi_{\mathrm{e}}$, and arrays used by the solver routine. The memory usage was approximately $0.5 \mathrm{kB}$ per node.

Equation (9) only determines $\phi_{\mathrm{e}}$ up to an additive constant, so that the linear system is singular. Singularity is by itself not a problem for Krylov-subspace methods such as BICGSTAB. However, for large systems such as ours, accumulated rounding errors can build up a residual component in the direction of the eigenvector associated with the singularity, i.e. the common mode of the extracellular potentials. This component can spoil the solver's estimate of convergence. A similar problem occurs when the system is made nonsingular by setting the potential at one or more nodes to zero. Therefore we adapted the BICGSTAB routine to explicitly orthogonalize the residual with respect to the common mode, and did not fix any potentials. A reference potential taken from the roof of the right atrium was subtracted from the extracellular potentials after a solution was obtained.

The membrane potential $V_{\mathrm{m}}$ was computed with a time step of $\delta t=10 \mu \mathrm{s}$. The stepsize was doubled when all cells finished depolarization. Depolarization was assumed to occur when the activation gate $m$ of the Na current [34] reached the value of 0.98 . The stepsize for $V_{\mathrm{m}}$ computation was limited both by the time constants of the $\mathrm{Na}$ current and by the stability criterion determined by the conductivities and spatial discretization [14], [43]. As proposed by Vigmond et al., the computation of $\phi_{\mathrm{e}}$ was performed less frequently [25], [38]. This is advantageous because the iterative solution of (9) is much more time-consuming and more difficult to parallelize than the straightforward evaluation of (8). We generally updated $\phi_{\mathrm{e}}$ once in every $5 \delta t$, i.e. at $50-\mu$ s intervals during depolarization and $100-\mu$ s intervals during repolarization.

\section{Comparison with a monodomain model}

The monodomain model can be derived from the bidomain model by assuming that the "monodomain conductivity tensors" $G_{\mathrm{i}}^{\prime}$ and $G_{\mathrm{e}}^{\prime}$ satisfy $G_{\mathrm{i}}^{\prime}=(1+\xi) G^{\prime}$ and $G_{\mathrm{e}}^{\prime}=(1+\xi) / \xi G^{\prime}$ 
where $\xi$ is a scalar and $G^{\prime}$ is the "bulk conductivity tensor" [7], [10] whose elements are

$$
G_{\mu \nu}^{\prime}=\frac{G_{\mathrm{i} \mu \nu} G_{\mathrm{e} \mu \nu}}{G_{\mathrm{i} \mu \nu}+G_{\mathrm{e} \mu \nu}}
$$

By substituting $G_{\mathrm{i}}^{\prime}$ and $G_{\mathrm{e}}^{\prime}$ for $G_{\mathrm{i}}$ and $G_{\mathrm{e}}$ in (7), a linear relation between $\phi_{\mathrm{e}}$ and $V_{\mathrm{m}}$ is obtained:

$$
\phi_{\mathrm{e}}=-\frac{\xi}{1+\xi} V_{\mathrm{m}}+\phi_{\mathrm{off}}
$$

where $\phi_{\text {off }}$ is a constant offset potential. If this expression for $\phi_{\mathrm{e}}$ is substituted in (6), an R-D equation is obtained in which $\phi_{\mathrm{e}}$ does not occur:

$$
\frac{\partial V_{\mathrm{m}}}{\partial t}=\frac{1}{\beta C_{\mathrm{m}}}\left\{\nabla \cdot\left(G^{\prime} \nabla V_{\mathrm{m}}\right)-\beta\left(I_{\text {ion }}+I_{\mathrm{s}}\right)\right\}
$$

Since $\phi_{\mathrm{e}}$ plays no role in the monodomain model, there is no implicit equation to solve. This makes monodomain models much less demanding than bidomain models.

We performed monodomain simulations by computing propagation of $V_{\mathrm{m}}$ from (16) instead of (6). In both monodomain and bidomain simulations, $\phi_{\mathrm{e}}$ was computed from (7), i.e., based on the bidomain conductivity tensors $G_{\mathrm{i}}$ and $G_{\mathrm{e}}$. For the monodomain model, this corresponds to solving a separate forward problem, and is only done once per millisecond of simulated time. In the monodomain model, the propagation velocity is expected to be proportional to the square root of the bulk conductivity [49]. We computed $G^{\prime}$ using (14) from the conductivities in Tab. I. Thus, the bulk conductivity was the same in our monodomain and bidomain simulations, so propagation patterns should match exactly if the monodomain approximation were valid.

\section{E. Simulations}

Simulations using varying numbers of processors for short intervals during depolarization were used to measure the scalability of the program, i.e. its ability to use a larger number of processors efficiently. In the same way, the performance of the two preconditioners was evaluated.

Propagation velocities were measured in a $40 \times 40 \times 40 \mathrm{~mm}$ tissue block with straight fibers, which had a $1 \mathrm{~cm}$-thick fluid bath on top and was insulated on the 5 remaining sides. The block was stimulated in the center of the tissue volume. Due to symmetry only a quarter of the block had to be computed. This simulation was also performed with a reduced spatial step $(\delta x)$ of $0.1 \mathrm{~mm}$, and with both bidomain and monodomain models. Another test of the model was performed by simulating epicardial maps of extracellular potentials obtained after epicardial and intramural pacing and comparing them with experimental results obtained in dogs by Taccardi et al. [30].

Finally, we compared activation times, action potential (AP) durations, AP waveforms, and extracellular potentials between monodomain and bidomain simulations using 1) a model with intracavitary blood, 2) a model with only a thin layer of blood on the ventricular walls, and 3) a model with no fluid at all. This comparison was performed for the epicardial pacing experiment described above, as well as for a sinus beat.

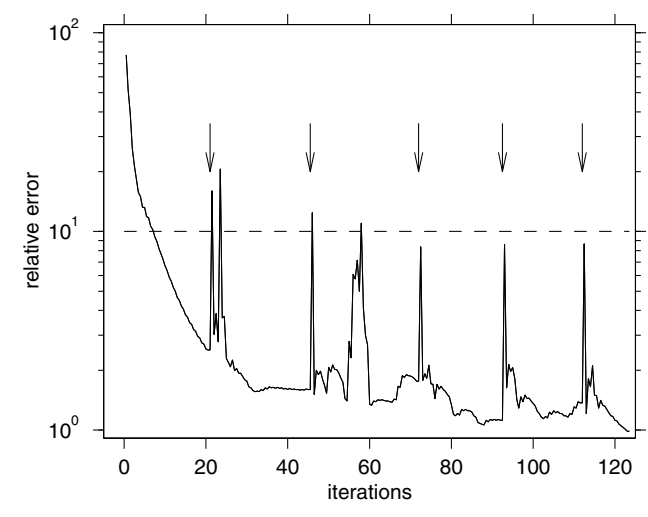

Fig. 3. Example of the convergence behavior of the solver for $\phi_{\mathrm{e}}$. The error is given relative to the tolerance level $L_{2}$ used for output of signals. The higher tolerance level $L_{1}$ used at intermediate time steps is indicated with a dashed line. This level falls in the relatively fast initial convergence regime. Gray vertical lines indicate restarts of the BiCGStab routine. The first restart is preceded by a short interval of stagnation. Each restart allows further convergence at the price of an initial divergence.

\section{RESULTS}

\section{A. Program behavior}

The heart model at $0.2-\mathrm{mm}$ resolution had 26 million nodes representing active tissue, for which $V_{\mathrm{m}}$ and $\phi_{\mathrm{e}}$ were computed, and 10 million nodes representing connective tissue and atrial muscle, for which only $\phi_{\mathrm{e}}$ was computed. Simulations with the resulting 36-million-node "dry heart" model produced $\phi_{\mathrm{e}}>40 \mathrm{mV}$ in late-activated areas. We considered this to be beyond the physiological range [30], [50], [51]. Therefore we performed all following simulations with a 1-mm layer of fluid appended to the endocardial and epicardial surfaces, a situation approximating a Langendorff setup. This measure added 9 million nodes for which $\phi_{\mathrm{e}}$ was computed. Filling the cavities with blood resulted in another 10 million such nodes. Thus, we computed 26 million membrane potentials and either 45 million or 55 million extracellular potentials. These models used $22 \mathrm{~GB}$ and $26 \mathrm{~GB}$ of memory, respectively.

Fig. 3 illustrates the typical convergence behavior of the solver for $\phi_{\mathrm{e}}$. Initially the convergence was relatively fast and regular. A subsequent slowdown coincided with a loss of accuracy in one of the minimization parameters in the solver [45]. A restart of the solver made further convergence possible but introduced an irregular convergence regime. Two tolerance levels, $L_{1}$ and $L_{2}$, were used; $L_{1}$ is indicated with a dashed line in the figure. Use of $L_{1}$ resulted in $\phi_{\mathrm{e}}$ that were accurate enough for correct computation of $V_{\mathrm{m}}$. Comparison with a simulation using a 100 times lower tolerance level showed differences in activation time of at most $0.01 \mathrm{~ms}$ and differences in $\phi_{\mathrm{e}}$ of at most $1.5 \mathrm{mV}$. Differences in $V_{\mathrm{m}}$ were in the order of $0.02 \mathrm{mV}$, except during the steep AP upstroke, where they could be as large as $3 \mathrm{mV}$ during one sample.

Electrograms computed with $L_{1}$, however, were not satisfactory. They typically featured depression of the S-T segment to about $-1 \mathrm{mV}$, and had values around $+1 \mathrm{mV}$ at $t=600 \mathrm{~ms}$, where they are expected to be zero. These offsets were proportional to the tolerance level. We decided to use a level $L_{2}=0.1 L_{1}$ for computation of $\phi_{\mathrm{e}}$ just before output (once 
(a)

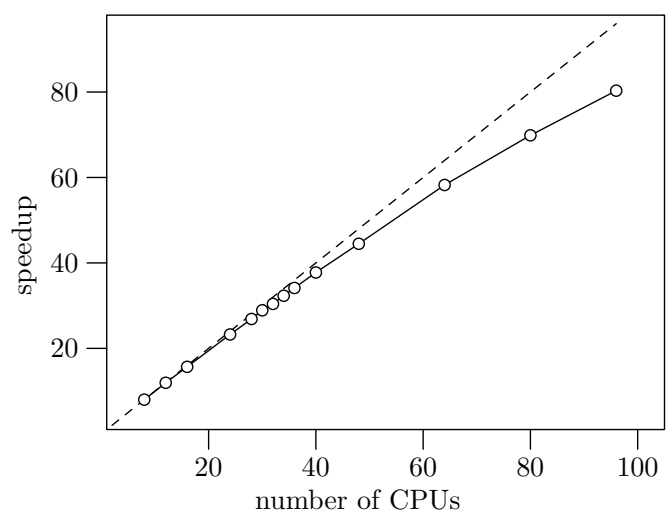

(b)

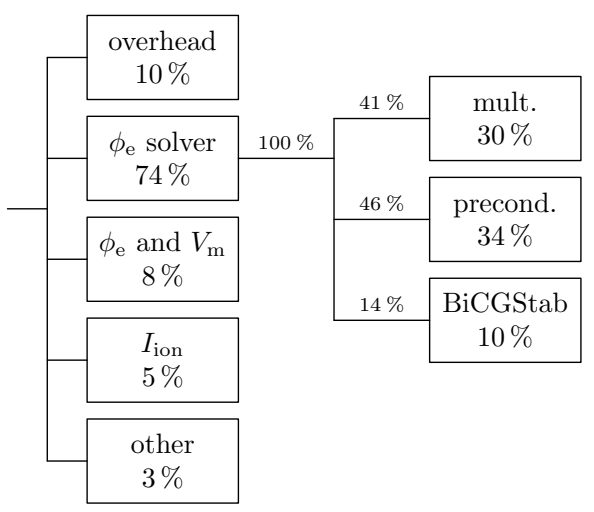

Fig. 4. (a) Speedup as a function of the number of processors. The speedup for $N$ processors is defined as the runtime with one processor divided by the runtime with $N$ processors. We used a minimum of 8 processors. The speedup is therefore computed as 8 times the runtime with 8 processors divided by the runtime with $N$ processors. The dashed line indicates ideal speedup.

(b) Relative time consumption of the major program components for a simulation with 55 million nodes on 32 processors. The code that solves the extracellular potential $\phi_{\mathrm{e}}$ is by far the most time-consuming, using $74 \%$. It is subdivided into "multiplier," the code that multiplies the problem matrix by a given vector; (ILU) preconditioner; and "BiCGStab," the solver proper, which uses little time compared to the multiplier and the preconditioner. After the solver, parallelization overhead (including load imbalance) is the most time-consuming with $10 \%$. Other computations for $V_{\mathrm{m}}$ and $\phi_{\mathrm{e}}$ use $8 \%$, the membrane model takes $5 \%$, and the remainder of the program $3 \%$.

per ms). Since the application of $L_{2}$ increased the number of iterations by a factor 2.5 on average, we used $L_{1}$ for all other updates of $\phi_{\mathrm{e}}$ (once per $5 \delta t$, i.e. 19 times per ms during depolarization).

Simulation of one heart beat $(600 \mathrm{~ms})$ in sinus rhythm took 39 hours with empty ventricles and 51 hours with the bloodfilled version on 32 processors. Fig. 4(a) shows the speedup obtained with various numbers of processors for a heart model of 45 million nodes. We had to use at least 8 processors, to obtain the memory required by the program. Speedup for 8 processors is defined as 8 . For the case of 32 processors, the number that we usually employ, the speedup is then 30.6 , corresponding to a performance loss of $4 \%$. The loss grows to $8 \%$ for 64 processors and $16 \%$ for 96 processors, the maximum that we could use for this test. Fig. 4(b) shows how time consumption, measured in CPU cycles, was divided over the major program components.

The performance of the two preconditioners was compared for a simulation of a heart model of 55 million nodes with
TABLE II

PROPAGATION VELOCITIES

\begin{tabular}{lccc}
\hline & $\delta x(\mathrm{~mm})$ & longitudinal & transverse \\
\hline bidomain & 0.10 & 770 & 278 \\
monodomain & 0.10 & 756 & 277 \\
bidomain & 0.20 & 731 & 218 \\
monodomain & 0.20 & 713 & 217 \\
\hline
\end{tabular}

32 processors. The diagonal preconditioner was 18 times faster $(0.02 \mathrm{~s}$ per call) than the ILU preconditioner $(0.36 \mathrm{~s})$. For the solver as a whole the ratio is much closer to unity due to other time-consuming routines (Fig. 4b), which took a total of $0.76-0.78 \mathrm{~s}$ per iteration. The preconditioner is called twice per iteration [45]. Thus, a single solver iteration took $0.88 \mathrm{~s}$ with the diagonal preconditioner versus $1.50 \mathrm{~s}$ with the ILU preconditioner. However, the number of iterations necessary for the depolarization phase was 3.7 times larger with the diagonal preconditioner than with the ILU preconditioner. Consequently, the total runtime was 2.0 times larger with the diagonal preconditioner.

\section{B. Simulation results}

Propagation velocities were measured in a $40 \times 40 \times 20 \mathrm{~mm}$ tissue block covered with a $10 \mathrm{~mm}$ fluid. This simulation was also performed with a reduced spatial step $(\delta x)$ of $0.1 \mathrm{~mm}$, and with both bidomain and monodomain models. Results are shown in Tab. II. Use of a spatial resolution of $0.2 \mathrm{~mm}$ led to longitudinal and transverse velocities that were $95 \%$ and $78 \%$, respectively, of the values obtained with $0.1-\mathrm{mm}$ resolution. With $0.1-\mathrm{mm}$ resolution, the ratio of longitudinal to transverse velocity was 2.8 ; with $0.2-\mathrm{mm}$ resolution it was 3.4. The longitudinal velocity was $2 \%$ higher in the bidomain than in the monodomain simulations, at both resolutions.

A verification of the model was performed by simulating epicardial potential maps obtained after epicardial and intramural pacing in open-chest dogs [30]. Development of the potential pattern reflected the transmural rotation of fibers, showing an expansion and counterclockwise rotation of the positive areas for (sub)epicardial pacing, expansion and clockwise rotation for subendocardial pacing, and a more symmetric expansion for mid-wall pacing. The case of sub-epicardial pacing is illustrated in Fig. 5.

The two maxima in the pattern had slightly different magnitudes, as was also observed experimentally [30]. In order to determine if the difference in the maxima was purely a projection effect, with one maximum appearing smaller just because it lies deeper in the wall, we inspected isopotential surfaces, and found that the larger maximum was actually stronger and surrounded by a larger region of positive potentials. We quantified the strength of the maxima by integrating, for each of the two positive regions, the potential values over the volume where $\phi_{\mathrm{e}}>0.1 \mathrm{mV}$. The resulting value was consistently about $20 \%$ larger for one of the maxima, throughout the 40-ms interval that we simulated. Extracellular potentials calculated from monodomain $V_{\mathrm{m}}$ were nearly identical to bidomain 


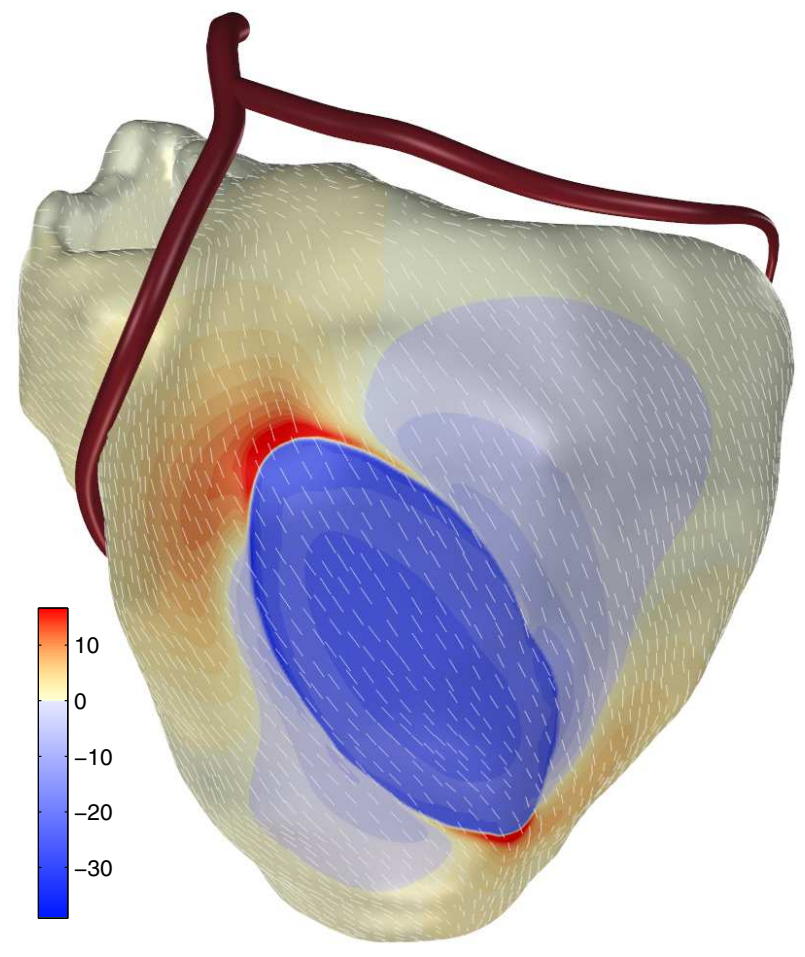

Fig. 5. Extracellular potentials on the left ventricular epicardial surface $28 \mathrm{~ms}$ after subepicardial pacing. Only the ventricular muscle is shown, with the left anterior descending and left circumflex arteries for orientation. Short white line segments indicate epicardial fiber orientation. A nearly elliptic dark-blue area indicates the negative extracellular potentials that are due to depolarized tissue; deviations from an elliptic shape are caused by the counterclockwise transmural fiber rotation. Low-level negative potentials also witness this rotation. Positive potentials (yellow to red) occur where depolarization travels along the fibers. The curved positive area, which can be well distinguished on the top left side, is again due to the fact that this longitudinal direction is rotated counterclockwise in deeper layers.

results. All characteristics that were mentioned, including the difference in maxima, were reproduced by $\phi_{\mathrm{e}}$ computed from monodomain $V_{\mathrm{m}}$.

For a sinus beat, total depolarization of the ventricles took $103.5 \mathrm{~ms}$ in the monodomain model, $98.4 \mathrm{~ms}$ in the dry heart, $97.1 \mathrm{~ms}$ in the heart with fluid layer, and $97.0 \mathrm{~ms}$ in the heart with filled cavities. Activation time (AT) was defined as the instant where the activation gate $m$ of the Na current [34] reached the value of 0.98 , and was measured with $0.01-\mathrm{ms}$ accuracy. The difference in AT between the two models, as a function of AT in the bidomain model, grew almost linearly throughout the depolarization phase.

Simulated electrograms are shown in Fig. 6. These signals may be compared with measured electrograms. Fig. 6 demonstrates features, such as low-amplitude complexes in the RV basal area, small $\mathrm{R}$ waves (initial positive waves) and large $\mathrm{S}$ (negative) waves in the middle part of the RV free wall, and large $\mathrm{R}$ waves on the left ventricular wall, that can be observed on the human heart [50], [52]. The figure also shows that differences between monodomain and bidomain models are small compared to differences between empty and filled ventricles.

Repolarization time (RT) was defined as the earliest instant where $V_{\mathrm{m}}=-70 \mathrm{mV}$ and $d V_{\mathrm{m}} / d t<0$. Action potential duration (APD) was defined as RT minus AT. In the model with filled cavities the APD was lengthened by 1 to $1.5 \mathrm{~ms}$ in the area that was activated in the first $10 \mathrm{~ms}$. This effect cannot be reproduced by the monodomain model, in which there is no representation of intracavitary fluid. Since the APD differences were very small, the RT differences were governed by the AT differences described above.

Minima and maxima of extracellular potentials throughout the heart were considered as a function of time. The range was beyond $(-60,+40) \mathrm{mV}$ in the dry model. It was $(-53,+29) \mathrm{mV}$ in the model with fluid layer and $(-44,+36) \mathrm{mV}$ in the fluid-filled model. Maximum potentials, reflecting $\mathrm{R}$-waves, were generally higher at later points in time, but the extrema did not grow monotonically. This demonstrates that both global electrotonic effects and local fiber orientation contribute to R-wave magnitude. For the monodomain model the ranges were $(<-60\rangle+40,),(-54,+30)$, and $(-44,+34)$, respectively.

Filling of the cavities reduced R-wave amplitude within $4 \mathrm{~mm}$ of the endocardial surface by a few millivolts, and increased R-wave amplitude in midmural and epicardial layers by up to $10 \mathrm{mV}$ (Fig. 6). These effects too were accurately reproduced by $\phi_{\mathrm{e}}$ computed from monodomain $V_{\mathrm{m}}$. Potentials in the intracavitary fluid were always negative during depolarization, even very close to the endocardium.

\section{DISCUSSION}

\section{A. Bidomain versus Monodomain}

The bidomain model is widely used for simulation of propagation in tissue preparations [53]-[55]. More recently, its application was extended to hearts of small mammals: Trayanova et al. described a finite-element model of the rabbit heart with $0.5-\mathrm{mm}$ resolution [25]. Henriquez et al. reported a $75 \mu \mathrm{m}$-resolution finite-volume model of the mouse heart [24]. The small size of these hearts made it possible to use a high-resolution grid while keeping the number of nodes well below a million. Simulations in tissue preparations have used several millions of nodes [56], [57], and simulation of the human heart requires a grid size that is almost two orders of magnitude larger. Lines et al. [26] have discussed techniques for a bidomain torso-coupled human heart model, but have shown only example simulations with a very coarse grid. With currently available hardware we had to resort to a resolution of $0.2 \mathrm{~mm}$ for our FD model of a human heart, comprised of 26 million tissue nodes and up to 29 million nodes in the surrounding fluid. Our model simulated $600 \mathrm{~ms}$ in a heart with blood-filled ventricles in about 2 days on 32 processors.

We have investigated in this paper the impact of the monodomain assumption on simulated propagation in an isolated human heart, by comparing results with a bidomain model. Differences between the two models were extremely small, even if extracellular potentials were influenced considerably by fluid-filled cavities. There was a small difference in propagation velocity between the monodomain and the bidomain model. All other properties of the membrane potentials and extracellular potentials simulated by the bidomain model were accurately reproduced by the monodomain model with a 


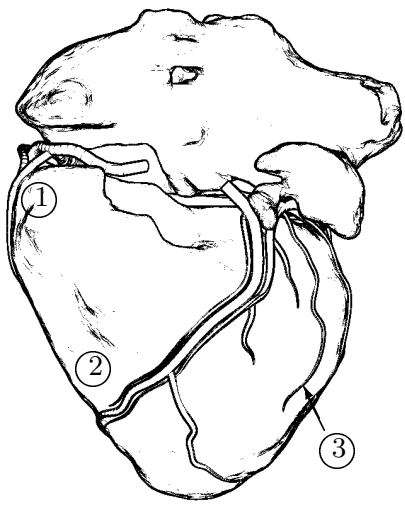

1

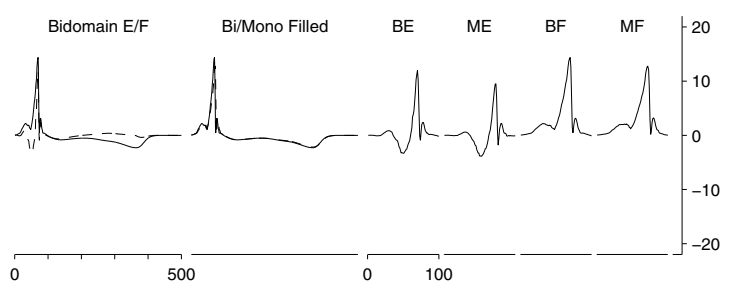

2
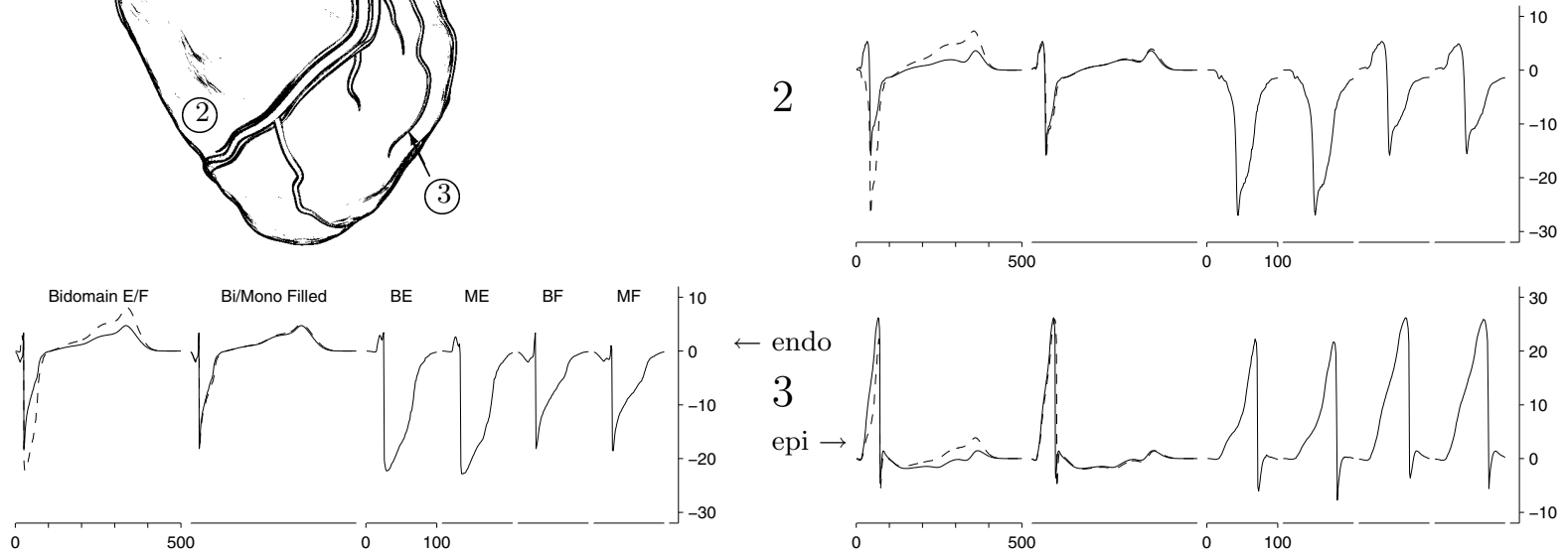

Fig. 6. Examples of extracellular potentials (electrograms) obtained with a monodomain and forward model and with a bidomain model, at 3 locations on the epicardial surface of the ventricles, and one on the endocardial surface. Electrograms were obtained either in ventricles with only a fluid layer ("Empty") or with filled cavities ("Filled"). For each site, 6 frames of axes are shown. The first compares empty (gray line) with filled ventricles (black line) in a bidomain simulation. The second frame compares monodomain (gray) with bidomain results (black) for the case of filled ventricles. The remaining four frames show the first $100 \mathrm{~ms}$ of the electrogram in empty bidomain (BE); empty monodomain (ME); filled bidomain (BF); and filled monodomain (MF) simulations.

forward solver for the extracellular potential. We have repeated the experiments shown in this paper with simulated ischemia and with the Na conductivity [34] reduced to $1 / 10$ of its normal value, and arrived at the same conclusions.

Since the monodomain approximation is not even approximately valid (Tab. I) it may be expected that monodomain simulation results differ importantly from bidomain results. Monodomain R-D models were developed before the first bidomain R-D models, and few papers have compared monodomain with bidomain results. Those that did, have shown small differences [38], and monodomain simulations have provided realistic results [10]-[14], [16]. The epicardial potential results of Taccardi et al. were accurately reproduced by bidomain, monodomain, and eikonal models [15], [58], [59]. The success of the monodomain approximation may be considered a common wisdom, which we have formalized by demonstrating that even if differences between monodomain and bidomain results can be found, they are small enough to be ignored for most applications, with the obvious exception of simulations involving applied currents. Another exception must be made with respect to the influence of adjacent fluid on $V_{\mathrm{m}}$ within a few hundred $\mu \mathrm{m}$ from the surface [9], [21]. These effects seem to be ignorable on the scale of a human heart. However, they may be significant in hearts of small rodents, and are critically important for the understanding of superfused preparations [60]. We attribute the success of monodomain models not to the validity of the assumption of equal anisotropy (which results in unrealistic extracellular potentials), but rather to the observation that the difference between monodomain and bidomain $\phi_{\mathrm{e}}$ is a smooth function of position. This difference therefore contributes little to the diffusion current.
By itself, a monodomain model does not produce realistic extracellular potentials. Especially in the in-vivo human heart, extracellular potentials are the primary means to study the tissue from nearby, using endocardial catheters and epicardial electrodes. Extracellular potentials may be computed from the membrane currents produced by a monodomain model. Such a solution is able to account for the effect of the thorax and the intracavitary blood on $\phi_{\mathrm{e}}$ [5]. If no applied currents are to be simulated, this method suffices if it is assumed that extracardiac conductivities have no significant influence on the source. We have shown that the $\phi_{\mathrm{e}}$ thus computed are very close to those of a bidomain model.

The method to solve the forward problem for our monodomain model is the same as in the bidomain model. While this provides a fair comparison, it is not a typical example of a forward model. Such models usually employ a few dozen current dipoles [4], [5], or epicardial potentials sampled at a few hundred points [61], [62]. Our "source model," in contrast, incorporated membrane potentials throughout the heart sampled at $0.2-\mathrm{mm}$ resolution. We did not investigate the impact of lower-resolution source representations in this study.

The conductivity of the thorax has been shown to influence epicardial $\phi_{\mathrm{e}}$ [22], [62]-[64]. However, its influence on $V_{\mathrm{m}}$ was never assessed. Modeling studies establishing the influence of torso coupling have used epicardial $\phi_{\mathrm{e}}$ as the source model [22], [62]. Experimental studies have shown that epicardial $\phi_{\mathrm{e}}$ changes [63], [64], but effects on $V_{\mathrm{m}}$ were not reported. A source model consisting of membrane currents may be more stable since the membrane can be thought of as a current source. With respect to the isolated heart, we have 
shown that intracavitary conductivity changes $\phi_{\mathrm{e}}$, but not $V_{\mathrm{m}}$. We have verified that this change in $\phi_{\mathrm{e}}$ can be computed from monodomain $V_{\mathrm{m}}$ just by employing an appropriate forward model. The conductivity of the thorax may be expected to have a larger influence on cardiac potentials than the intracavitary blood. Therefore it would be useful to verify the applicability of the monodomain assumption for the in-situ heart. This requires coupling of the heart to a torso model. Torso coupling was described by several groups [22], [23], [26], but never applied to a complete high-resolution 3-D human heart and torso. To implement a thoracic coupling for our heart model we would have to use a discretization method that allows a coarser grid at some distance from the heart. While this is feasible, it is somewhat challenging to reconcile with the streamlined solver routines that we currently employ to make bidomain modeling on the scale of a human heart at all possible.

While the differences between monodomain and bidomain results are small enough to permit the use of a monodomain model for propagation studies, there is no doubt that a bidomain model is necessary to simulate pacing or defibrillation. These topics were not addressed in this study. Our model can be used to some extent to simulate pacing with extracellularly applied currents. However, defibrillation currents would cause erroneous activations in an FD model with irregular boundaries. Our methods can be used for defibrillation only in rectangular blocks aligned with the coordinate axes.

The time gain of using a monodomain model and a separate forward model to compute $\phi_{\mathrm{e}}$ results from solving $\phi_{\mathrm{e}}$ less often (e.g. 1/ms) than in a bidomain R-D model, and perhaps on a coarser grid [38]. This gain is limited because a lower tolerance level is needed for computation of electrograms than for propagation of potentials per se. Solving $\phi_{\mathrm{e}}$ on the same grid as used in the bidomain model, at 1-ms intervals, our monodomain and forward model were only about twice as fast as our bidomain model.

\section{B. Epicardial mapping}

The epicardial mapping data reported by Taccardi et al. constitute an interesting challenge to computer heart models [30]. Several researchers have attempted to simulate these results [15], [58], [59], and have explained many of the reported features. An exception is the observed difference in strength of the two maxima in the potential patterns, which Taccardi and colleagues attributed to the imbrication angle of the fibers [30]. Despite the presence of imbrication in their model of an idealized ventricle, Colli Franzone et al. did not obtain an important difference [15]; but Muzikant et al. [65] did, using a realistic imbrication angle of $5^{\circ}$. We studied simulated epicardial potentials following subepicardial stimulation and observed asymmetry in the positive potentials even when imbrication was zero. We hypothesize that this difference is due to varying wall thickness or slight convergence/divergence of the fiber orientations, but we presently did not investigate these hypotheses.

\section{The model}

Due to its relatively low spatial resolution of $0.2 \mathrm{~mm}$, our model underestimates the transverse propagation velocity by about $22 \%$ for the parameters chosen in this study. This may be compensated by assuming a larger conductivity, but more elegant solutions may also be conceived. First, more accuracy can be obtained with a higher resolution, which we currently cannot implement because it would require too much computer memory and CPU time. Second, a higherorder estimate for the diffusion operator $\nabla \cdot\left(G_{\mathrm{i}} \nabla\right)$ in (6) or an integral equation for the diffusion current [8] could be employed. These would increase memory usage and runtime less. Third, a finite-element or finite-volume method may be more accurate at the same or even a coarser discretization [25], [57]. Finally, we could use a modification of the membrane model that slows down the depolarizing sodium current to make the depolarization wavefront thicker and less steep [66]. The side effects of such modifications are hard to predict, so this method should be used with caution when membrane behavior is studied.

The value of $\beta=1000 \mathrm{~cm}^{-1}$ used in this study is low compared to measured values [67]. Together with the bulk conductivity and the membrane capacitance $C_{\mathrm{m}}, \beta$ determines propagation velocity. Values used in modeling studies vary from 200 to 3000 [8], [9], [12], [14], [18], [22], [24], [25], [34], [67]-[69]. Generally, authors of propagation studies who reported realistic propagation velocities have found it necessary to use low values of $\beta$. A possible explanation for this discrepancy is that due to the tight packing of fibers into bundles, effective $\beta$ or $C_{\mathrm{m}}$ is smaller in tissue than in isolated myocytes, in which they are usually measured.

We used a mathematical definition of fiber orientation that is conceptually simple, but is able to represent the known macroscopic features such as alignment with the surfaces and transmurally rotating fiber orientation. Regional variations in the range of transmural rotation can be made, although presently we only assigned different ranges to the left and right ventricles. This mathematical definition is an improvement of the methods used previously by Lorange et al. [14], [27] and is based on qualitative and quantitative knowledge of cardiac anatomy [29], [31]. Several groups have reported the use of fiber orientations measured by histological means or by diffusion tensor imaging [70]-[72]. We have not used such data because we feared that measurement errors might occasionally cause discontinuities and large imbrication angles, which would be difficult to repair. In addition, our mathematical definition facilitates study of recognizable features, such as imbrication, in isolation.

The linear system solver and preconditioner used are among the most powerful algorithms of their kind. However, recent comparisons suggest that multilevel methods could be competitive [73], [74]. Our model readily solved the small-scale features of $\phi_{\mathrm{e}}$, which are of importance for the propagation algorithm. It had difficulty to reach the lower tolerance level $L_{2}$ because it could not easily resolve smaller gradients, which build up appreciable errors over long distances-especially in large models. Multilevel methods should be able to deal better with the long-distance features, and would thus be especially advantageous if correct $\phi_{\mathrm{e}}$ traces are required, rather than accurate $\nabla \cdot\left(G_{\mathrm{i}} \nabla \phi_{\mathrm{e}}\right)$ for use in (6).

In this study we used a membrane model that is a compro- 
mise between accuracy of representation and computational efficiency [34]. However, the model is designed to work with any membrane model, can assign different membrane models to different nodes, and can employ spatially varying parameters for the membrane model. Since the membrane routine takes a relatively small fraction of the computation time, we have not considered additional optimizations for the membrane model such as adaptive time steps [41], [75].

The memory usage of the program, $0.5 \mathrm{kB}$ per node, compares favorably to the estimate of at least $2 \mathrm{kB}$ per node given by Lines et al. [26]. This is due to several savings. We used a regular mesh, for which no geometrical data and very few topological data need to be stored. In addition, we found that the matrices $A$ and $B$ as well as the ILU coefficients can be stored in single precision. For single-beat simulations, the membrane state variables too can be stored in single precision. Finally, we used a relatively simple membrane model with only 6 state variables.

The speedup (runtime reduction when more processors are employed) of our model is satisfactory. Although we optimized our program for use with 16 to 32 processors, speedup from 32 to 64 processors is a factor 1.92. This implies a performance loss of only $4 \%$. Thus, it will be relatively easy to improve the spatial resolution of our model when larger equipment becomes available for our work.

\section{CONCLUSiON}

We have developed a bidomain R-D model of the human heart that can compute a full cardiac cycle in a reasonable period of time. The model can also operate as a monodomain, optionally with a forward solver for $\phi_{\mathrm{e}}$. We have shown that a bidomain model is not necessary for propagation studies. Thus, even with the (more economical) monodomain model we can simulate epicardial, endocardial, intracavitary, and intramyocardial signals that can be compared with measured signals. Both models can be used to study the changes that occur in these signals as a result of abnormalities in ionic currents and in tissue properties.

\section{ACKNOWLEDGMENT}

The work described in this paper was initiated under the direction of Dr. Ramesh M. Gulrajani, who passed away unexpectedly while the manuscript was still an early draft. We gratefully dedicate this paper to our deceased colleague.

We would like to thank the editors and the anonymous reviewers for their thoughtful comments and suggestions, Éric Bélanger for the initial implementation of the BICGSTAB solver, and several colleagues for proofreading our manuscript. Computations were performed at the Réseau québécois de calcul de haute performance (RQCHP).

\section{REFERENCES}

[1] C. S. Henriquez, "Simulating the electrical behavior of cardiac tissue using the bidomain model," CRC Crit. Rev. Biomed. Eng., vol. 21, pp. 1-77, 1993.

[2] O. H. Schmitt, "Biological information processing using the concept of interpenetrating domains," in Information Processing in The Nervous System, K. N. Leibovic, Ed. New York: Springer, 1969, pp. 325-331.
[3] L. Tung, "A bi-domain model for describing ischemic myocardial D-C potentials," Ph.D. dissertation, MIT, Cambridge MA, USA, 1978.

[4] W. T. Miller, III and D. B. Geselowitz, "Simulation studies of the electrocardiogram; I. The normal heart," Circ. Res., vol. 43, no. 2, pp. 301-315, 1978.

[5] R. M. Gulrajani and G. E. Mailloux, "A simulation study of the effects of torso inhomogeneities on electrocardiographic potentials, using realistic heart and torso models," Circ. Res., vol. 52, pp. 45-56, 1983.

[6] W. T. Miller, III and D. B. Geselowitz, "Simulation studies of the electrocardiogram; II. ischemia and infarction," Circ. Res., vol. 43, no. 2, pp. 315-323, 1978.

[7] R. M. Gulrajani, Bioelectricity and Biomagnetism. New York, NY: Wiley, 1998.

[8] R. C. Barr and R. Plonsey, "Propagation of excitation in idealized anisotropic two-dimensional tissue," Biophys. J., vol. 45, pp. 1191-1202, 1984.

[9] B. J. Roth, "Action potential propagation in a thick strand of cardiac muscle," Circ. Res., vol. 68, pp. 162-173, 1991.

[10] L. J. Leon and B. M. Horáček, "Computer model of excitation and recovery in the anisotropic myocardium. I. Rectangular and cubic arrays of excitable elements," J. Electrocardiol., vol. 24, no. 1, pp. 1-15, 1991.

[11] R. Hren and B. M. Horáček, "Value of simulated body surface potential maps as templates in localizing sites of ectopic activation for radiofrequency ablation," Physiol. Meas., vol. 18, pp. 373-400, 1997.

[12] G. Huiskamp, "Simulation of depolarization in a membrane-equationsbased model of the anisotropic ventricle," IEEE Trans. Biomed. Eng., vol. 45 , no. 7 , pp. $847-855,1998$.

[13] O. Bernus, B. van Eyck, H. Verschelde, and A. V. Panfilov, "Transition from ventricular fibrillation to ventricular tachycardia: a simulation study on the role of $\mathrm{Ca}^{2+}$-channel blockers in human ventricular tissue," Phys. Med. Biol., vol. 47, pp. 4167-4179, 2002.

[14] M.-C. Trudel, B. Dubé, M. Potse, R. M. Gulrajani, and L. J. Leon, "Simulation of propagation in a membrane-based computer heart model with parallel processing," IEEE Trans. Biomed. Eng., vol. 51, no. 8, pp. 1319-1329, 2004.

[15] P. Colli-Franzone, L. Guerri, and B. Taccardi, "Modeling ventricular excitation: axial and orthotropic anisotropy effects on wavefronts and potentials," Math. Biosci., vol. 188, pp. 191-205, 2004

[16] O. Berenfeld and S. Abboud, "Simulation of cardiac activity and the ECG using a heart model with a reaction-diffusion action potential," Med. Eng. Phys., vol. 18, no. 8, pp. 615-625, 1996.

[17] B. Rodríguez, L. Li, J. C. Eason, I. R. Efimov, and N. A. Trayanova, "Differences between left and right ventricular chamber geometry affect cardiac vulnerability to electric shocks," Circ. Res., vol. 97, pp. 168-175, 2005.

[18] G. Plank, L. J. Leon, S. Kimber, and E. J. Vigmond, "Defibrillation depends on conductivity fluctuations and the degree of disorganization in reentry patterns," J. Cardiovasc. Electrophysiol., vol. 16, pp. 205-216, 2005

[19] T. Ashihara and N. A. Trayanova, "Asymmetry in membrane responses to electric shocks: Insights from bidomain simulations," Biophys. J., vol. 87, pp. 2271-2282, 2004.

[20] I. R. Efimov, R. A. Gray, and B. J. Roth, "Virtual electrodes and deexcitation: New insights into fibrillation induction and defibrillation," J. Cardiovasc. Electrophysiol., vol. 11, pp. 339-353, 2000.

[21] C. S. Henriquez, A. L. Muzikant, and C. K. Smoak, "Anisotropy, fiber curvature, and bath loading effects on activation in thin and thick cardiac tissue preparations: Simulations in a three-dimensional bidomain model," J. Cardiovasc. Electrophysiol., vol. 7, pp. 424-444, 1996.

[22] M. L. Buist and A. J. Pullan, "The effect of torso impedance on epicardial and body surface potentials: A modeling study," IEEE Trans. Biomed. Eng., vol. 50, no. 7, pp. 816-824, 2003.

[23] G. Fischer, B. Tilg, R. Modre, G. J. M. Huiskamp, J. Fetzer, W. Rucker, and P. Wach, "A bidomain model based BEM-FEM coupling formulation for anisotropic cardiac tissue," Ann. Biomed. Eng., vol. 28, pp. 1229-1243, 2000.

[24] C. S. Henriquez, J. V. Tranquillo, D. Weinstein, E. W. Hsu, and C. R. Johnson, "Three-dimensional propagation in mathematic models: Integrative model of the mouse heart," in Cardiac Electrophysiology; From Cell To Bedside, 4th ed., D. P. Zipes and J. Jalife, Eds. Philadelphia, PA: Saunders, 2004.

[25] N. Trayanova and F. Aguel, "Computer simulations of cardiac defibrillation: a look inside the heart," Comput. Vis. Sci., vol. 4, pp. 259-270, 2002 .

[26] G. T. Lines, M. L. Buist, P. Grøttum, A. J. Pullan, J. Sundnes, and A. Tveito, "Mathematical models and numerical methods for the forward 
problem in cardiac electrophysiology," Comput. Vis. Sci., vol. 5, pp. 215239, 2003.

[27] M. Lorange and R. M. Gulrajani, "A computer heart model incorporating anisotropic propagation: I. Model construction and simulation of normal activation," J. Electrocardiol., vol. 26, no. 4, pp. 245-261, 1993.

[28] I. Ragnemalm, "Neighborhoods for distance transformations using ordered propagation," CVGIP: Image Understanding, vol. 56, no. 3, pp. 399-409, 1992.

[29] D. D. Streeter, Jr, H. M. Spotnitz, D. P. Patel, J. Ross, and E. H. Sonnenblick, "Fiber orientation in the canine left ventricle during diastole and systole," Circ. Res., vol. 24, pp. 339-347, 1969.

[30] B. Taccardi, E. Macchi, R. L. Lux, P. R. Ershler, S. Spaggiari, S. Baruffi, and Y. Vyhmeister, "Effect of myocardial fiber direction on epicardial potentials," Circulation, vol. 90, pp. 3076-3090, Dec. 1994.

[31] R. Beyar and S. Sideman, "A computer study of the left ventricular performance based on fiber structure, sarcomere dynamics, and transmural electrical propagation velocity," Circ. Res., vol. 55, pp. 358-375, 1984.

[32] G.-R. Li, J. Feng, L. Yue, and M. Carrier, "Transmural heterogeneity of action potentials and $I_{\text {to1 }}$ isolated from the human right ventricle," Am. J. Physiol. Heart Circ. Physiol., vol. 275, pp. H369-H377, 1998.

[33] E. Drouin, F. Charpentier, C. Gauthier, K. Laurent, and H. Le Marec, "Electrophysiologic characteristics of cells spanning the left ventricular wall of human heart: Evidence for presence of M cells," J. Am. Coll. Cardiol., vol. 26, pp. 185-192, 1995.

[34] O. Bernus, R. Wilders, C. W. Zemlin, H. Verschelde, and A. V. Panfilov, "A computationally efficient electrophysiological model of human ventricular cells," Am. J. Physiol. Heart Circ. Physiol., vol. 282, pp. H2296-H2308, 2002.

[35] P. G. A. Volders, K. R. Sipido, E. Carmeliet, R. L. H. M. G. Spätjens, H. J. J. Wellens, and M. A. Vos, "Repolarizing $\mathrm{K}^{+}$currents $I_{\mathrm{TO} 1}$ and $I_{\mathrm{Ks}}$ are larger in right than left canine ventricular midmyocardium," Circulation, vol. 99, pp. 206-210, 1999.

[36] J. Di Diego, J. Cordeiro, R. J. Goodrow, J. M. Fish, A. C. Zygmunt, G. Pérez, F. Scornik, and C. Antzelevitch, "Ionic and cellular basis for the predominance of the Brugada syndrome phenotype in males," Circulation, vol. 106, pp. 2004-2011, 2002.

[37] D. Durrer, R. T. van Dam, G. E. Freud, M. J. Janse, F. L. Meijler, and R. C. Arzbaecher, "Total excitation of the isolated human heart," Circulation, vol. 41, no. 6, pp. 899-912, 1970.

[38] E. J. Vigmond, F. Aguel, and N. A. Trayanova, "Computational techniques for solving the bidomain equations in three dimensions," IEEE Trans. Biomed. Eng., vol. 49, no. 11, pp. 1260-1269, 2002.

[39] B. J. Roth, "Electrical conductivity values used with the bidomain model of cardiac tissue," IEEE Trans. Biomed. Eng., vol. 44, pp. 326-328, 1997.

[40] H. I. Saleheen and K. T. Ng, "New finite difference formulations for general inhomogeneous anisotropic bioelectric problems," IEEE Trans. Biomed. Eng., vol. 44, no. 9, pp. 800-809, 1997.

[41] B. Victorri, A. Vinet, F. A. Roberge, and J.-P. Drouhard, "Numerical integration in the reconstruction of cardiac action potentials using Hodgkin-Huxley-type models," Comp. Biomed. Res., vol. 18, pp. 10-23, 1985.

[42] S. Rush and H. Larsen, "A practical algorithm for solving dynamic membrane equations," IEEE Trans. Biomed. Eng., vol. 25, no. 4, pp. 389-392, 1978.

[43] W. H. Press, S. A. Teukolsky, W. T. Vetterling, and B. P. Flannery, Numerical Recipes in C; The Art of Scientific Computing, 2nd ed. Cambridge, UK: Cambridge University Press, 1992.

[44] P. Sonneveld, "CGS, a fast Lanczos-type solver for nonsymmetric linear systems," SIAM J. Sci. Stat. Comput., vol. 10, pp. 35-52, 1989.

[45] H. A. van der Vorst, "Bi-CGSTAB: A fast and smoothly converging variant of Bi-CG for the solution of nonsymmetric linear systems," SIAM J. Sci. Stat. Comput., vol. 13, pp. 631-644, 1992.

[46] Y. Saad, Iterative Methods for Sparse Linear Systems. New York: PWS publishing, 1996, second edition published by SIAM, Philadelphia, 2003.

[47] T. F. Chan, E. Gallopoulos, V. Simoncini, T. Szeto, and C. H. Tong, "A quasi-minimal residual variant of the Bi-CGStab algorithm for nonsymmetric systems," SIAM J. Sci. Comput., vol. 15, no. 2, pp. 338347, 1994.

[48] R. C. Penland, D. M. Harrild, and C. S. Henriquez, "Modeling impulse propagation and extracellular potential distributions in anisotropic cardiac tissue using a finite volume element discretization," Comput. Visual. Sci, vol. 4, pp. 215-226, 2002.

[49] P. Colli-Franzone, L. Guerri, and C. Viganotti, "Oblique dipole layer potentials applied to electrocardiology," J. Math. Biol., vol. 17, pp. 93124, 1983.
[50] J. P. Roos, R. T. van Dam, and D. Durrer, "Epicardial and intramural excitation of normal heart in six patients 50 years of age and older," $\mathrm{Br}$. Heart J., vol. 30, pp. 630-637, 1968.

[51] M. Potse, P. F. H. M. van Dessel, A. C. Linnenbank, N. M. van Hemel, C. A. Grimbergen, and J. M. T. de Bakker, "Properties of unipolar electrograms recorded with a multielectrode basket catheter," J. Electrocardiol., vol. 37, pp. 1-10, 2004.

[52] M. Biermann, M. Shenasa, M. Borggrefe, G. Hindricks, W. Haverkamp, and G. Breithardt, "The interpretation of cardiac electrograms," in Cardiac Mapping, 2nd ed., M. Shenasa, M. Borggrefe, and G. Breithardt, Eds. Oxford, UK: Blackwell, 2003, ch. 2.

[53] K. Gima and Y. Rudy, "Ionic current basis of electrocardiographic waveforms; A model study," Circ. Res., vol. 90, pp. 889-896, 2002.

[54] J. M. Fish and C. Antzelevitch, "Cellular and ionic basis for the sexrelated difference in the manifestation of the Brugada syndrome and progressive conduction disease phenotypes," J. Electrocardiol., vol. 36 Suppl., pp. 173-179, 2003.

[55] M. Buist, G. Sands, P. Hunter, and A. Pullan, "A deformable finite element derived finite difference method for cardiac activation problems," Ann. Biomed. Eng., vol. 31, pp. 577-588, 2003.

[56] R. Weber dos Santos, G. Plank, S. Bauer, and E. J. Vigmond, "Parallel multigrid preconditioner for the cardiac bidomain model," IEEE Trans. Biomed. Eng., vol. 51, no. 11, pp. 1960-1968, 2004.

[57] M. Trew, I. Le Grice, B. Smaill, and A. Pullan, "A finite volume method for modeling discontinuous electrical activation in cardiac tissue," Ann. Biomed. Eng., vol. 33, no. 5, pp. 590-602, 2005.

[58] A. L. Muzikant and C. S. Henriquez, "Paced activation mapping reveals organization of myocardial fibers: A simulation study," J. Cardiovasc. Electrophysiol., vol. 8, pp. 281-294, 1997.

[59] A. E. Pollard, M. J. Burgess, and K. W. Spitzer, "Computer simulations of three-dimensional propagation in ventricular myocardium; effects of intramural fiber rotation and inhomogeneous conductivity on epicardial activation," Circ. Res., vol. 72, no. 744-756, 1993.

[60] B. J. Roth, "Influence of a perfusing bath on the foot of the cardiac action potential," Circ. Res., vol. 86, pp. e19-22, 2000.

[61] R. C. Barr, M. Ramsey, III, and M. S. Spach, "Relating epicardial to body surface potential distributions by means of transfer coefficients based on geometry measurements," IEEE Trans. Biomed. Eng., vol. 24, no. 1 , pp. $1-11,1977$.

[62] R. N. Klepfer, C. R. Johnson, and R. S. MacLeod, "The effects of inhomogeneities and anisotropies on electrocardiographic fields: A 3D finite-element study," IEEE Trans. Biomed. Eng., vol. 44, no. 8, pp. 706-719, 1997.

[63] L. S. Green, B. Taccardi, P. R. Ershler, and R. L. Lux, "Epicardial potential mapping: Effects of conducting media on isopotential and isochrone distributions," Circulation, vol. 84, pp. 2513-2521, Dec. 1991.

[64] R. S. MacLeod, B. Taccardi, and R. L. Lux, "The influence of torso inhomogeneities on epicardial potentials," in Computers in Cardiology. Piscataway, NJ: IEEE Press, 1994, pp. 793-796.

[65] A. L. Muzikant, E. W. Hsu, P. D. Wolf, and C. S. Henriquez, "Region specific modeling of cardiac muscle: Comparison of simulated and experimental potentials," Ann. Biomed. Eng., vol. 30, pp. 867-883, 2002.

[66] O. Bernus, H. Verschelde, and A. V. Panfilov, "Modified ionic models of cardiac tissue for efficient large scale computations," Phys. Med. Biol., vol. 47, no. 11, pp. 1947-1959, 2002.

[67] C.-H. Luo and Y. Rudy, "A dynamic model of the cardiac ventricular action potential; I. Simulations of ionic currents and concentration changes," Circ. Res., vol. 74, no. 6, pp. 1071-1096, jun 1994.

[68] G. T. Lines, P. Grøttum, and A. Tveito, "Modeling the electrical activity of the heart; A bidomain model of the ventricles embedded in a torso," Comput. Vis. Sci., vol. 5, pp. 195-213, 2003.

[69] A. L. Muzikant and C. S. Henriquez, "Bipolar stimulation of a threedimensional bidomain incorporating rotational anisotropy," IEEE Trans. Biomed. Eng., vol. 45, no. 4, pp. 449-462, 1998.

[70] I. J. Legrice, P. J. Hunter, and B. H. Smaill, "Laminar structure of the heart: a mathematical model," Am. J. Physiol. Heart Circ. Physiol., vol. 272, pp. H2466-H2476, 1997.

[71] M. F. Beg, P. A. Helm, E. McVeigh, M. I. Miller, and R. L. Winslow, "Computational cardiac anatomy using MRI," Magn. Res. Med., vol. 52, pp. 1167-1174, 2004.

[72] L. Geerts, P. Bovendeerd, K. Nicolay, and T. Arts, "Characterization of the normal cardiac myofiber field in goat measured with MR-diffusion tensor imaging," Am. J. Physiol. Heart Circ. Physiol., vol. 283, pp. H139-H145, 2002.

[73] M. Mohr and B. Vanrumste, "Comparing iterative solvers for linear systems associated with the finite difference discretisation of the forward 
problem in electro-encephalographic source analysis," Med. Biol. Eng.

Comput., vol. 41, pp. 75-84, 2003.

[74] T. M. Austin, M. L. Trew, and A. J. Pullan, "Solving the cardiac bidomain equations for discontinuous conductivities," IEEE Trans. Biomed. Eng., vol. 53, no. 7, pp. 1265-1272, 2006.

[75] M. D. Lesh, M. Pring, and J. F. Spear, "Cellular uncoupling can unmask dispersion of action potential duration in ventricular myocardium. A computer modeling study." Circ. Res., vol. 65, no. 5, pp. 1426-1440, 1989. 